\title{
DETECTING GROUPS OF EQUIDISTANT FREQUENCIES IN SPECTRA OF GEOMAGNETIC PULSATIONS
}

\author{
A.R. Polyakov \\ Institute of Solar-Terrestrial Physics SB RAS, \\ Irkutsk, Russia,polar@isz.irk.ru
}

\begin{abstract}
This paper presents a revised version of the new signal processing method based on the analysis of a specially constructed correlation function of amplitude and phase fluctuations (APCF). This method allows us to detect the presence of a group of equidistant frequencies in the spectrum of the original signal and to measure the difference of two adjacent frequencies $\Delta f$ in such a group. The end product of the processing is a histogram of a set of $\Delta f$. The effect of noise that may be present in the original signal has been examined. It has been shown that even with a very high level of noise when its component in the spectrum completely absorbs and masks spectral peaks of equidistant frequencies of the desired signal, the APCF method copes with the problem of detecting these frequencies. This method was first applied to processing of natural signals, for which recordings of geomagnetic field disturbances of an ultralow-frequency (ULF) range were used. The comparison of one of the histograms with the traditional spectrum indicates that the chaotic spectrum, which has always been considered to be a noise spectrum, actually
\end{abstract}

has a strictly ordered structure. It has been found that most spectral peaks belong to one of the sets (more than 10) of equidistant frequency groups. In the entire spectrum, peaks of these groups are superimposed on each other and form a complex chaotic sequence. The analysis of peaks of all histograms allows us to conclude that the equidistant frequency groups, which correspond to peaks in each histogram, are eigenfrequencies of the 2D Alfvén wave resonator. The existence of such a resonator in the magnetosphere in the vicinity of the outer edge of the plasmapause has been predicted in theoretical studies [Guglielmi, Polyakov, 1983; Leonovich, Mazur, 1987]. The APCF processing method enables us to experimentally confirm this prediction.

Keywords: signal processing technique, correlation functions, eigenfrequencies.

\section{INTRODUCTION}

This paper is a sequel of [Polyakov, 2010; Polyakov, 2014, 2017], which formulated and developed a new original method for analyzing the harmonic structure of broadband oscillations. The proposed processing method is not associated with spectral analysis. It is based on the analysis of a specially constructed correlation function of amplitude and phase fluctuations (APCF), which are transformed components of an original signal. Further details of the processing method are discussed in the next section.

In [Polyakov, 2010; Polyakov, 2014], an essential fundamental feature of APCF has been identified using examples of the processing of oscillations of waves of different types in 1D- and 2D resonators. When a group of equidistant frequencies appears in the original signal spectrum, plots of APCF against $\tau$ reveal peaks following each other at regular intervals. In these studies, a universal empirical formula has been derived which relates these intervals to the difference of two adjacent frequencies of an equidistant group. It became obvious that the analysis of APCF peaks with software tools enables us to detect groups of equidistant frequencies in a broadband signal spectrum.

The next paper of this squeal [Polyakov, 2017] has proposed a new more efficient algorithm of the first stage of the processing (see Section 1), which makes the new APCF method and processing program more versa- tile. Now we can use this method to handle any original discrete signals, for which the Fourier transform is valid.

In recent years there has been an advance in algorithms for the analysis of peaks of correlation functions. The computer program has been completed at last. Processing of simulated signals has shown that all updates have improved the program. The final result in all cases has a much smaller statistical dispersion.

The above papers focused on solving methodological problems. Now, when these studies are nearing completion and we have an almost ready-made method and a corresponding computer program, a logical step is to begin processing real natural signals. It is necessary to ensure that in this case the proposed method will provide reliable results and to compare them with the results of the traditional spectral analysis. It is the main purpose of this paper. As real signals I have chosen time records of geomagnetic disturbances in the ultralowfrequency range on the dayside magnetosphere.

Real oscillations as opposed to simulated ones very often contain random noise of natural or anthropogenic origin. Before processing, we should therefore figure out how much the presence of noise can distort the results. Results of this study are presented in Section 2.

\section{PROCESSING METHOD}

As mentioned above, this paper uses an updated program; the updates apply not only to the latest proce- 
dures that determine the presence of periodically recurring peaks in correlation functions. The previous version of the program is described in detail in [Polyakov, 2017]. Here I will briefly repeat this description, paying particular attention to the recent updates. Just as in [Polyakov, 2017], all procedures are divided into three groups. Each group may be regarded as one of the processing stages, which yields a certain intermediate result.

\subsection{Filtration transform}

The original signal $x\left(t_{i}\right)$ should be given in a discrete form: $t_{i}=(i-1) \Delta t ; i=1,2, \ldots, N$, where $\Delta t$ is the time step. The only applicability condition for the APCF method is a large array of values - at least $N \sim 50$ thousand, better 100-200 thousand.

The technique for transforming an original signal at the first stage is similar to the digital filtration using the Fourier transform. It is described in detail in [Polyakov, 2017] and has since remained unchanged.

The transformed signal is a sum of monochromatic oscillation and original signal with greatly reduced amplitude at all frequencies. On an oscillogram, it looks like a slightly noisy sine curve, in which monochromatic oscillations represent a mean line (in what follows they are referred to as mean oscillations). The original signal causes small deviations from this line.

The frequency of mean oscillations $f_{0}$ and the amplitude $A_{0}$ are specified in the program. During processing, three $f_{0}$ values are used. Being transformed, the original signal has a certain frequency range set in the program before processing. Amplitudes at all frequencies are approximately two orders of magnitude lower than $A_{0}$.

\subsection{Amplitude and phase fluctuations}

At the beginning of this stage, we specify the length of the short interval with the number of steps $N_{0}<<N$. This interval will be moved throughout the transformed quasi-monochromatic signal with a step $N_{0} / 5$. After each step, a new time fragment of this signal falls within this interval. All the following processing procedures are applied to each short fragment.

Determine amplitude and phase fluctuations. Convert the discrete signal values and time to a dimensionless form: $x_{i} \rightarrow x_{i} / A_{0} ; \quad t_{i} \rightarrow 2 \pi f_{0} t_{i}$.

At each step, calculate the derivative $y_{i}=d x_{i} / d t$, and plot the points $\left(x_{i}, y_{i}\right)$ on the surface of rectangular phase coordinates $x, \dot{x}$. The mean oscillation in these coordinates is a unit circle (mean cycle). For oscillations after the filtration transform, in view of the contribution made by small fluctuations, the cycles will be close to this circle. In the previous version [Polyakov, 2017], the mean oscillation was referred to [Gudzenko, 1962] as the average of all cycles. Now it is decided to abandon this procedure and consider the unit circle to be the mean cycle. This has almost no effect on results of the processing, but significantly reduces its duration.

Then, for each point $\left(x_{i}, y_{i}\right)$ estimate the deviation from the mean cycle along the normal direction (for the circle it is the direction along the radius) $n_{i}$ and the phase of the mean cycle $\Theta_{i}$ at the point of its intersection with the normal. On the phase plane, $\Theta_{i}$ coincides with the length of the path of a representative point along the mean cycle; and $t_{i}$ (in terms of normalization), with the path length along initial cycles. The phase difference $\gamma_{i}=\Theta{ }_{i}-t_{i}$ can therefore be regarded as a tangential deviation of the point from the mean cycle. For oscillations after the filtration transform normal and tangential deviations at each step $n_{i}\left(\Theta_{i}\right)$ and $\gamma_{i}=\left(\Theta_{i}\right)$ represent deviations of amplitude and phase from the amplitude and phase of the mean oscillation. Given that $\gamma_{i}<<1$, the phase of the mean oscillation $\Theta_{i}=2 \pi f_{0} t_{i}$, where $t_{i}$ is the unnormalized (real) time.

It must be emphasized that small deviations from the mean oscillation are caused solely by one original signal, so amplitude and phase fluctuations should be considered as two different independent components of the signal.

\subsection{Correlation functions}

At this stage, for the $n(\Theta), \gamma(\Theta)$ fluctuations, find cross-correlation and autocorrelation functions and substitute them into:

$$
\begin{aligned}
& G(\tau)=\overline{[\gamma(\Theta) \gamma(\Theta-\tau)]} \overline{[n(\Theta) n(\Theta-\tau)]}- \\
& -\overline{[\gamma(\Theta) n(\Theta-\tau)]} \overline{[n(\Theta) \gamma(\Theta-\tau)]} .
\end{aligned}
$$

Unlike [Polyakov, 2017], in this study each correlator marked with a bar depends only on the phase shift $\tau$, and for each of its values it is determined by averaging over the $\Theta$ phase, just as the traditional correlation function of original signal in radiophysical methods. In addition, (1) has one function rather than three. The other two were decided to omit because they contain derivatives of $n$ and $\gamma$ with respect to $\Theta$ in the correlators, which often lead to a significant scatter in the final processing results.

Function (1) will sometimes be called the correlation function of amplitude and phase fluctuations (APCF); and the technique for deriving and analyzing it, the APCF method. As noted in Introduction, the APCF dependence on phase shift $\tau$ has an important and unique feature.

In [Polyakov, 2010; Polyakov, 2014], a vast number of APCF obtained from the oscillation processing in numerical models of resonators (1D and 2D) and waveguides for waves of different types (elastic waves [Polyakov, 2010], fast magnetosonic (FMS) waves, and Alfvén waves with regard to transverse dispersion [Polyakov, 2014]) have been analyzed. It has been established that in all the cases without exception the $G(\tau)$ dependence is a periodic sequence of narrow peaks. It has also been shown that these peaks are directly related to resonator or waveguide eigenfrequencies. This relationship is analogous to the principle of operation of the optical diffraction grating [Polyakov, 2017] - it is the same as the relationship between grating slits (eigenfrequencies) and spectral lines on a screen $(G(\tau)$ peaks).

The results obtained in [Polyakov, 2017] allow an important clarification. It turns out that an analog of grating slits may be not only eigenfrequencies but also any other group of equidistant frequencies if this group 
is present in the original oscillation spectrum, such as frequencies of nonlinear oscillations or any arbitrarily specified equidistant frequencies in the time dependence determined by the sum of harmonic functions. In all the cases, $G(\tau)$ has the form of a periodic sequence of peaks.

Another important result of the previous works is the universal empirical formula that determines for $G(\tau)$ the position of the $k$ peak on the $\tau$-axis:

$$
\tau_{k}=2 \pi k f_{0} / \Delta f,
$$

where $\Delta f$ is the difference of two adjacent frequencies of the equidistant group in the signal spectrum. This formula is similar [Polyakov, 2017] to the well-known relation for determining the angular position of the spectral line of an optical diffraction grating. In APCF method, it is used to measure $\Delta f$ in the same way as its analogue is used in optics.

\subsection{Analyzing APCF peaks}

Return to procedures of the processing method. At this stage, for $G(\tau)$ positions of all the $\tau_{k}$ peaks are found. Then, a special procedure analyzes ratios of these positions. The analysis results allow us to omit all $\tau_{k}$ such that $k>1$. There remains only the first-order position. It is not necessary that $\tau_{1}$ is the only value. If the spectrum contains not one group but several groups of equidistant frequencies distinct from each other in $\Delta f$, the $G(\tau)$ function has several periodic sequences of peaks. The position of peaks within one sequence is determined by relation (2), where $\Delta f$ characterizes one of the frequency groups. In this situation, after analyzing the peaks, we obtain several values of $\tau_{1}$.

Substituting these values into (2) for the first order, we get: $\Delta f=2 \pi f_{0} / \tau_{1}$.

Recall that APCF and $\Delta f$ are determined for each short fragment of the signal after the filtration transform. These fragments are indicated by a sliding time window. Once it reaches the end of the signal, we proceed to the beginning of stage 1 (filtration transform), change the frequency $f_{0}$, and process all fragments with this frequency again. Then repeat this for the third time with another value of $f_{0}$.

At the end of all the procedures, we have a wide array of $\Delta f$ values. At this point, the APCF processing is over. The end product is a histogram of these values.

In the traditional spectrum, the presence of a peak at a certain frequency means that fluctuations of this frequency are a part of the original signal. In our spectrum (histogram), each peak corresponds not to one frequency but to a group of equidistant frequencies of oscillations in the original signal. The position of the peak on the horizontal axis gives us not a separate frequency but the difference of two adjacent frequencies characteristic of the whole group.

\section{NOISE EFFECT IN THE ORIGINAL SIGNAL}

In this Section, I analyze a situation where a signal, in addition to fluctuations in the group of equidistant frequencies, contains noise modeled by random white noise. The signal is represented here as a sum of two terms, the first of which is a deterministic time dependence determined by the sum of harmonic functions with equidistant frequencies. The second term is a random component. In a discrete form at the $i$ time step, signal values are defined by

$$
\begin{aligned}
& x_{i}=\sum_{m=1}^{30} \cos \left[2 \pi f_{m}(i-1)\right]+a F_{i} \\
& f_{m}=0.04151+0.00333(m-1),
\end{aligned}
$$

where $F_{i}$ is the number ranging from 0 to 1 , which is given by a random number generator at each step, $a$ is the scale factor. The fluctuation amplitude at all frequencies $f_{m}$ is the same and equal to 1 . The $f_{m}$ frequencies compose an equidistant group with $\Delta f=0.00333$. Note that all frequencies and their differences here, later in the text, and in plots are expressed in dimensionless units: $f \rightarrow f \Delta t$, where $\Delta t$ is the time step of the original discrete signal.

In this case, we are interested in fluctuations with high and low levels of noise, so signal (3) will be processed at different values of $a$.

Figure 1, $a$ presents the processing results for $a=0$ with no noise. The left and central panels show the spectrum and $G(\tau)$ function for one of the time fragments at $f_{0}=0.04$. In terms of signal normalization after the filtration transform, the spectrum $\left|x_{\omega}\right|$ (modulus of the complex Fourier transform) and the function $G$ become dimensionless, and the phase shift $\tau$ of the mean oscillation is expressed in radians.

Note that the spectrum is not one of the intermediate results of the APCF processing method. It is shown here to illustrate the connection between the equidistant frequency group in the signal and periodic peaks of $G$. In it, according to (3), we find peaks at $f_{m}$. The $G(\tau)$ plot displays a pronounced periodic sequence of peaks. This confirms once again the main results of the previous studies [Polyakov, 2010; Polyakov, 2014, 2017]. The only difference is that in our case we set the signal in the form of a complete time function, whereas in the above works it was obtained from the numerical simulation of standing waves.

The right panel of Figure 1, $a$ shows the resulting histogram of $\Delta f$. On the vertical axis, $n_{0}$ is the total number of all processed time fragments. Values of $\Delta f$ are expressed in dimensionless units. It is seen that all the frequency differences form a narrow peak such that $\Delta f=0.0033$. This value, which results from the processing, coincides with the difference of adjacent frequencies $f_{m}$ in original signal (3). This coincidence confirms in this case the reliability of the results.

Of special note is the statistical character of the end product of the APCF processing, which distinguishes it from the traditional spectrum. When processing one time fragment, $\Delta f$ can be obtained only if the spectrum of the fragment comprises a group of equidistant frequencies. If a set of frequency differences in a histogram forms a peak, this means that spectra of most fragments have equidistant groups with the same value of $\Delta f$. It is obvious that if the presence of the equidistant groups is statistically significant for the set of time 

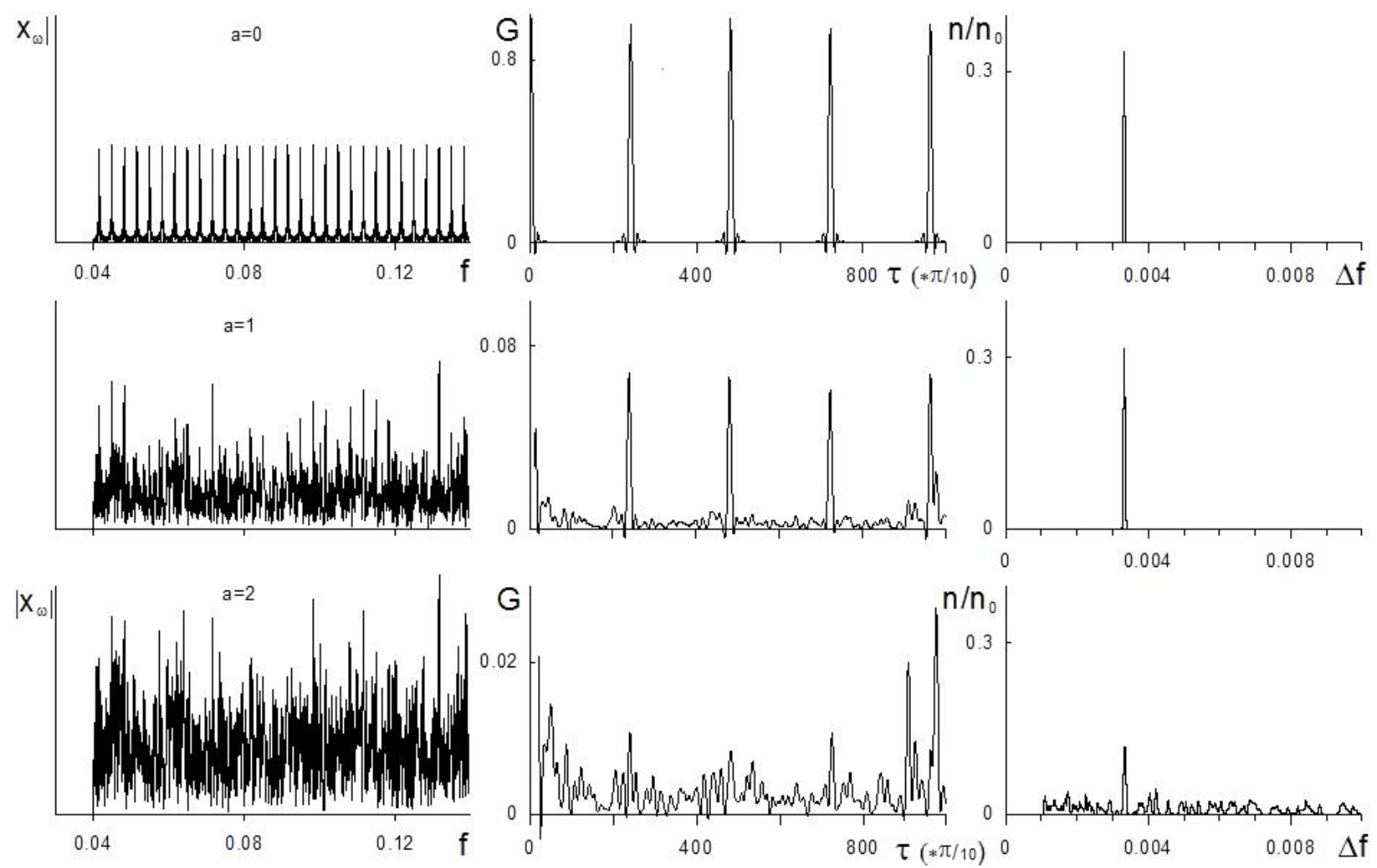

Figure 1. Dependence of processing results obtained by the APCF method on noise level in the original signal

fragments, the total spectrum of the original signal should contain the same frequency groups.

Return to original signal (3) and intensify noise: $a=1$. In this case, the total signal spectrum is a superposition of spectral components of the desired signal and noise. Figure $1, b$ (left panel) shows that the noise component makes a great contribution and significantly distorts the desired signal component. If we compare this spectrum with the spectrum in Figure 1, $a$, we can see that some peaks at $f_{m}$ almost completely disappeared. From the whole group of equidistant peaks throughout the frequency range there remain only individual fragments, and among them there occur noise component peaks comparable in height. At the same time, despite such significant distortions in the spectrum, characteristics of $G(\tau)$ peaks remain almost the same. If we compare them with the peaks in Figure 1, $a$ (central panel), it becomes apparent that the width and position on the $\tau$-axis for each peak are the same. The only significant change occurs in the height of the peaks - it decreases by almost an order of magnitude. However, since this height is not related to the procedure of determining the position of the first peak $\tau_{1}$ and the frequency difference, in the final histogram (right panel) we obtain a distinct peak with the same characteristics (width and position on the $\Delta f$ axis) as in the peak in the histogram of Figure 1, $a$.

Increase the noise level by a factor of two: $a=2$. In the spectrum in Figure 1, $c$, the noise component contribution becomes so great that it completely absorbs and masks peaks of equidistant frequencies. Among these peaks there occur many noise peaks, and we cannot keep track of which of them are equidistant. Moreover, if we know nothing about the origin of this spectrum, we cannot establish whether there are equidistant frequencies in it or not. In this case, changes in $G(\tau)$ are not so significant. In this function, we can clearly see the same peaks as in Figure $1, a, b$. A decrease in the height of the peaks does not affect their processing. There is a small number of new noise-related peaks, which should contribute to the final histogram. Indeed, in the histogram (right panel) there is the noise component contribution that is absent in the histograms of Figure $1, a, b$. We can also see that the peak in the frequency difference $\Delta f=0.00333$ is much higher than the noise peaks and is easily detected. By the presence of this distinct peak, we can confidently say that the chaotic spectrum (left panel) has equidistant frequencies with this difference of two adjacent frequencies.

In the APCF method, an interference for the group of equidistant frequencies in the signal is not only random noise frequencies but also any other alien frequencies such as frequencies of another equidistant group with another difference $\Delta f$ or frequencies of several such groups with different values of $\Delta f$. In the spectrum, all these frequencies form a complex chaotic structure of alternation of peaks, in which it becomes impossible to identify frequencies of each individual group. The above analysis of the processing results of signal (3) shows that the APCF method copes with this problem. It allows us to detect all groups of equidistant frequencies even if the signal has a complex chaotic spectrum. Moreover, the APCF method is designed for processing such signals. In such cases, we will be able to obtain new information that cannot be gained by traditional spectroscopy methods. 
As noted above, the APCF method enables us to detect equidistant frequency groups in initial oscillations and identify their characteristic difference of adjacent frequencies. This means that we can establish only the fact of the presence of these frequencies. We cannot find their specific values, as well as the total number of frequencies in the group. The generalization of the model signal processing results shows that the number of frequencies in a group is one of the factors determining the height of the $G(\tau)$ peaks. Another factor is noise frequencies (extraneous for a frequency group). If the number of frequencies in the group is small, with a high noise level we will have low peaks and in the final histogram we cannot get the maximum corresponding to this group. If we increase the number of frequencies in the group (e.g., for signal (3), this number is 30), the noise effect is overcome more effectively; the histogram displays a pronounced maximum as in Figure 1, $c$. The experience of model signal processing suggests that the number of frequencies in a group should be less than 5 . Only in this case with the noise level that is not very high, we can expect that the APCF method will detect such a group.

\section{RESULTS OF PROCESSING OF NATURAL SIGNALS}

In this section, I turn to the processing of signals from natural phenomena or objects. As such signals I consider time-dependent deviations of the geomagnetic field from the mean value in the $\mathrm{N}-\mathrm{S}$ direction. The data are presented in the discrete form, the time step is $0.1 \mathrm{~s}$. In the APCF computer program before the initial signal processing, a frequency range should be specified within which equidistant frequency groups are detected. In this case, this interval ranges from 0.02 to 0.22 (in dimensionless units). In hertz (0.2-2.2), this interval corresponds to the high-frequency component of the entire ULF range of geomagnetic disturbances.

Each original signal lasts for $1 \mathrm{hr}$. I have processed four signals recorded at Mondy Observatory $\left(\Phi=46.7^{\circ}\right.$, $\left.\Lambda=173.6^{\circ}, L=2.1\right)$ on April 10,2000 , where $\Phi$ and $\Lambda$ are corrected geomagnetic latitude and longitude, $L$ is the shell number. All the signals occurred in the dayside magnetosphere. Overall magnetospheric conditions correspond to the mean level with $K_{\mathrm{p}} \approx 3$.

A final result of the APCF processing for each hour signal is a histogram of $\Delta f$. One of them is shown in Figure 2, $a$, with universal and local time given. By comparison, Figure 2, $b$ depicts the traditional spectrum in the above frequency range. It is obvious that the histogram contains not a single peak, as shown in Figure 1, and not even two peaks but a whole set of pronounced, separated, and very high peaks. There are 11 such peaks in total, each with serial number in the plot.

The multiplicity of peaks in the histogram is a very interesting result. This should mean that the spectrum in Figure 2, $a$ has 11 groups of equidistant frequencies. Each group corresponds to one of the histogram peaks and features its own difference of two adjacent frequencies, which is determined by the position of the peak on the $\Delta f$ axis. Each group should comprise five frequen- cies. This is minimum estimate. In fact, since the peaks in the histogram are pronounced, it is more appropriate to have 10 frequencies in a group. It turns out here that in the spectrum in Figure 2, $a$, each of $\sim 110$ frequencies and peaks at them belong to a particular equidistant group. Different groups include most, if not all, peaks of the spectrum.

Peaks of each group, sequentially following each other, must cover a certain segment of the frequency axis. Obviously, fragments of different groups can at least partially, if not completely, overlap each other. As a result, we should observe a superposition of spectral peaks of different equidistant groups with different values of $\Delta f$, which involves not two and three groups of peaks but 11 groups. Hence, the spectrum has the high density of peaks that we can see in Figure 2, $a$. The analysis of the mutual arrangement of these peaks on the frequency axis is much more complicated and does not allow us to identify any regularities — we just see their chaotic alternation.

The spectrum similar to that shown in Figure 2, $a$ is generally considered to be the noise spectrum. It does not contain pronounced peaks at any individual frequencies and hence does not provide useful information. The APCF processing clearly shows that this spectrum has in fact a well-ordered structure of different frequency groups, which we can find in all other similar cases.

Figure 3 presents final histograms of $\Delta f$ for all processed hour signals. The local time of each of them is indicated on the plot. New histograms also demonstrate the same multiplicity of very clear, isolated peaks as the histogram in Figure 2, $b$ and Figure 3, $a$. In some of them, for example, in Figure $3 b, c$, there are even more peaks. This suggests that the spectra of all the processed signals have noise characteristics and should be similar in shape to the spectrum shown in Figure 2, $a$.

The analysis of Figure 3 indicates yet another important regularity. In each histogram, we can see that the gap between the peaks is almost constant with peak number. This is especially noticeable in Figure 3, $a$. In other cases, we can also assume that all the numbered peaks are equidistant. We encounter this feature again, but now it characterizes peaks in the histograms.

However, as seen in Figure 3, this feature can be seriously violated for peaks of some numbers. Without going into causes of these violations, note that the processing method permits a slight deviation of frequency differences $\Delta f$ from the true value. Sometimes it can reach $5 \%$. Since the average gap between the peaks is not very large, even minor shifts of the peaks can lead to a serious violation of equidistance.

For example, in Figure 3, c, peaks 7-10 are clearly not equidistant. However, if we take into account the possibility of shift of the peaks and shift peak 8 to the right and peak 9 to the left only by the width of each peak ( $5 \%$ of the $\Delta f$ peak is approximately equal to its width at the top), gap 8-9 significantly decreases, gaps $7-8$ and $9-10$ increase, and peaks $7-10$ become nearly equidistant. $3, b$. 


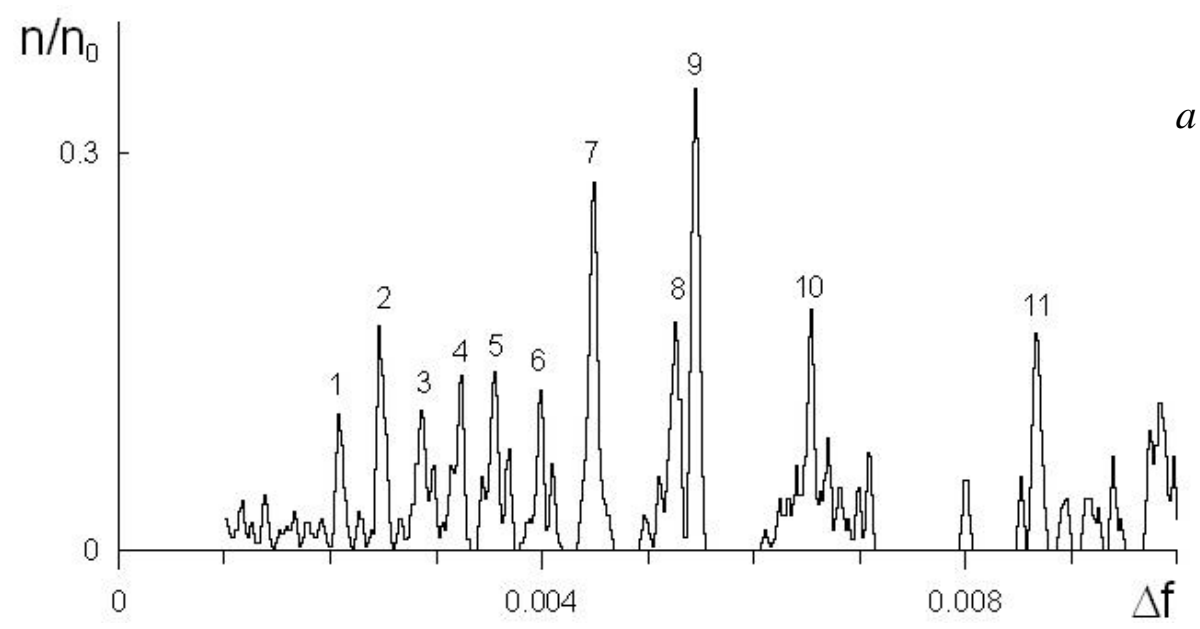

$\left|X_{\omega}\right|$

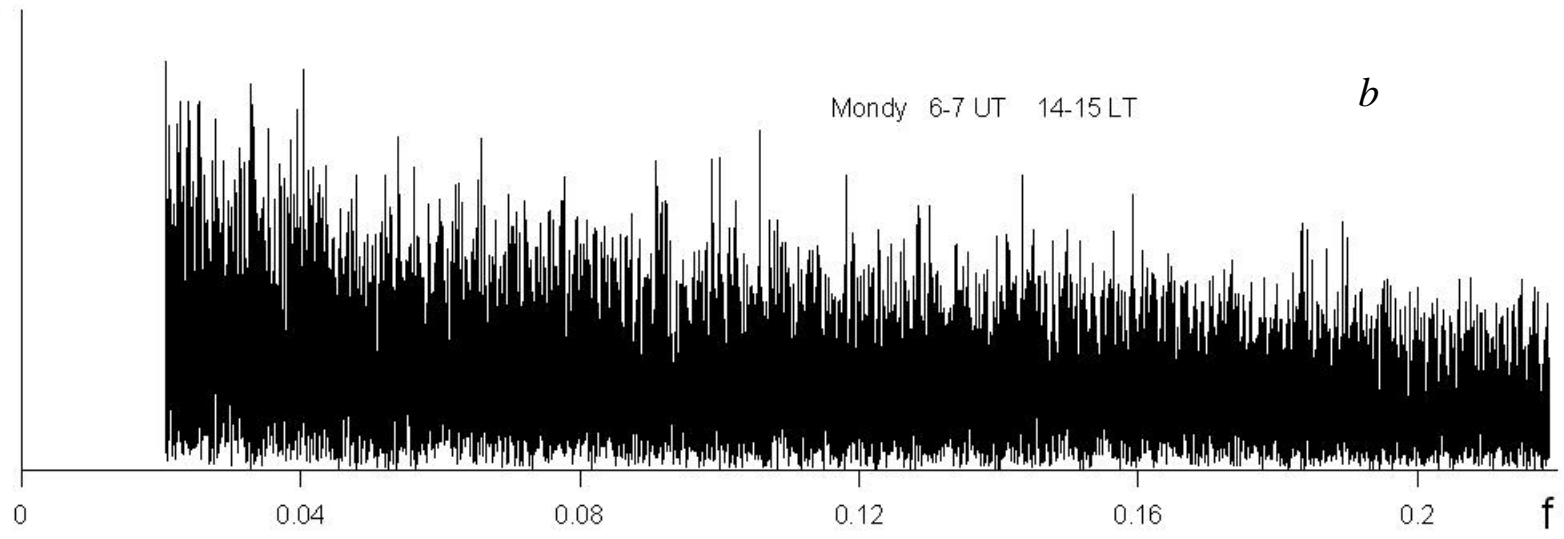

Figure 2. Histogram of $\Delta f$ (end product of the APCF processing) versus the traditional signal spectrum
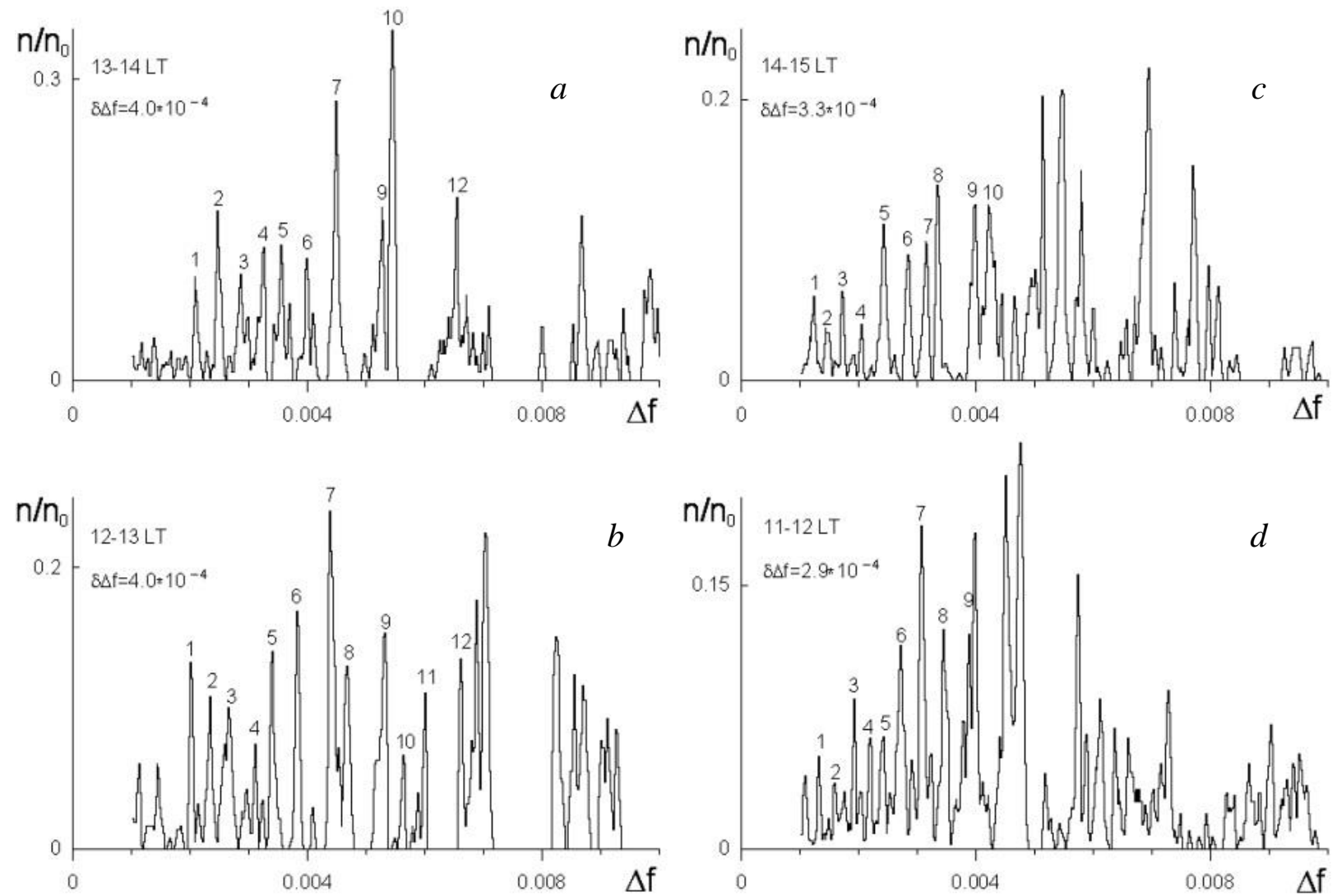

Figure 3. Histograms of $\Delta f$ for all processed natural signals 
For one standing Alfvén wave (field line resonance) all eigenfrequencies create a single peak. In [Polyakov, 2017], it has been shown that in the 2D FMS wave resonator, eigenfrequencies contain two equidistant groups. Each group is determined by frequencies of one of two possible 1D standing waves. Thus, frequencies of a 2D FMS standing wave can create two peaks in the histogram. The problem in this case is that within the entire dayside magnetosphere we cannot find a sufficient number of different standing FMS and Alfvén waves to use them to interpret all the numerous peaks in the histogram. In addition, we cannot expect that the $\Delta f$ parameters of eigenfrequencies of standing waves of different origin can be related by the equidistance condition. In this regard, let me call your attention to a resonator of MHD waves of another type, which can be seen in the dayside magnetosphere. It is the 2D Alfvén wave resonator. Eigenfrequencies of this resonator have an appropriate structure, which may be useful for interpreting peaks in histograms [Polyakov, 2017].

As for the Alfvén velocity distribution in different magnetospheric regions, it is known that in moving on the dayside from Earth along the radial coordinate, which enumerates magnetic shells, the Alfvén velocity decreases and reaches minimum at the inner edge of the plasmapause. At the plasmapause, the velocity increases more than by an order of magnitude and reaches a maximum at the outer edge. Behind the plasmapause in the outer magnetosphere, the velocity decreases again up to the magnetopause. The theoretical works [Guglielmi, Polyakov, 1983; Leonovich, Mazur, 1987] have shown that given the small transverse dispersion of Alfvén waves, in the vicinity of the maximum velocity at the outer edge there are favorable conditions for the occurrence of the $2 \mathrm{D}$ Alfvén wave resonator. Along the magnetic field, the waves are trapped, being reflected from conjugate ionospheres; and along the radial coordinate, they are reflected from walls of the potential well.

The dispersion relation in view of the transverse dispersion has the form

$$
\omega^{2}=k_{z}^{2} A^{2}\left(1+S^{2} k_{x}^{2}\right)
$$

where $A$ is the Alfvén velocity, $k_{z}$ and $k_{x}$ are the wave vector components along the direction of the magnetic field and radial coordinate, $S$ is the dispersion scale equal to the Larmor radius of radiation belt protons in the vicinity of the outer edge of the plasmapause.

Since I am interested only in the structure of eigenfrequencies of standing waves, it suffices to consider this problem qualitatively in a simplified form. Assume that in the vicinity of the outer edge of the plasmapause the magnetic field is direct and uniform. The plasma density and Alfvén velocity are also uniform. The existence of the 2D Alfvén wave resonator is due to the presence of reflecting walls, which trap it along the coordinates $z$ and $x$. Denote the respective distances between the walls by $l_{z}$ and $l_{x}$. In this case, the ratio for eigenvalues takes the form $k_{z}=\pi N / l_{z}$ and $k_{x}=\pi n / l_{x}$, where $N$ and $n$ are the harmonic numbers along $z$ and $x$. Substituting in (4), obtain an equation for eigenfrequencies

$$
\omega_{N n}=\frac{A \pi N}{l_{z}}\left(1+\frac{S^{2} \pi^{2} n^{2}}{l_{x}^{2}}\right)^{1 / 2}=\Omega_{N}\left(1+\frac{S^{2} \pi^{2} n^{2}}{l_{x}^{2}}\right)^{1 / 2},
$$

where $\Omega_{N}$ are the eigenfrequencies of longitudinal standing Alfvén waves (field line resonance).

To each number $N$ corresponds a frequency group with different numbers $n$. Since in this case $S<<l_{x}$, these frequencies are very densely fill the frequency axis and are difficult to detect in a signal, using the traditional spectrum. According to (5), given small numbers $n$, frequencies in a group are not equidistant. However, with increasing number up to $n>l_{x} /(\pi S),(5)$ is transformed into

$$
\omega_{N n}=\Omega_{N} \frac{S \pi n}{l_{x}},
$$

and the frequencies with different numbers $n$ become equidistant. Among all frequencies (5), each number $N$ has its equidistant group. The main characteristic of such a group - the difference of two adjacent frequencies - in dimensionless units is determined by

$$
\Delta f_{N}=\frac{\Delta t}{2 \pi}\left(\omega_{N, n+1}-\omega_{N, n}\right)=\frac{\Delta t}{2 \pi} \Omega_{N} \frac{S \pi}{l_{x}}=\frac{\Delta t}{T_{N}} \frac{S \pi}{l_{x}},
$$

where $T_{N}$ is the $N$ harmonic period.

Obviously, equidistant groups (6) and (7) of eigenfrequencies of the 2D Alfvén wave resonator are ideally suited for interpretation of the peaks in the histograms obtained by the APCF method. In this case, each peak corresponds to frequency group (6) with its number $N$. The number of peaks coincides with the total number of harmonics along the magnetic field, which are excited in the resonator. In Figure 3, b, for example, the set of peaks consecutive without gaps corresponds to the continuous sequence of numbers $N$. In one plot, we can see not 2-6 consecutive resonator harmonics but 12 ones.

Leonovich and Mazur [2016] have shown that in actual magnetospheric conditions (dipole magnetic field, plasma density and Alfvén velocity distribution) the $\Omega_{N}$ frequencies of both toroidal and poloidal modes become almost equidistant from the first harmonic number. From (7) for the position of the peak on the horizontal axis of the histogram it follows that $\Delta f_{N}=b \Omega_{N}$, where $b$ is the fixed factor. This means that peaks along the $\Delta f$ axis should be equidistant. We can see this feature of the peaks in all the histograms of Figure 3. The difference in positions of two adjacent peaks is found using (7):

$$
\begin{aligned}
& \delta \Delta f_{N}=\Delta f_{N+1}-\Delta f_{N}= \\
& =\frac{\Delta t}{2 \pi}\left(\Omega_{N+1}-\Omega_{N}\right) \frac{S \pi}{l_{x}}=\frac{\Delta t}{T_{1}} \frac{S \pi}{l_{x}},
\end{aligned}
$$

where $T_{1}$ is the period of the first harmonic. Given equidistance of the frequencies $\Omega_{N}, \Omega_{N 1}-\Omega_{N}=\Omega_{1}$. Experimental values of the mean interval between peaks (8) have been calculated for all histograms. The results in dimensionless units are presented in plots.

Pay attention to the features of the 2D Alfvén wave resonator structure along the radial coordinate. Its inner wall results from a sharp increase in the Alfvén velocity and is located at the plasmapause. Along the azimuthal coordinate, it moves by rotating to- 
gether with the plasmasphere. In this case, at the average geomagnetic activity this wall is located on the shell $L \approx 4$, and on the dayside magnetosphere it should be stable in all azimuthal coordinates, i.e. at any local time.

The outer wall stems from a decrease in the Alfvén velocity outside the plasmasphere. It does not rotate with Earth and should be fixed with respect to the magnetosphere. If we use averaged or smoothed values of plasma density to determine the Alfvén velocity, its decrease from a maximum at the outer edge to a minimum at the plasmapause is not very large (2-3 times), having a spatial scale equal to the radial size of the entire outer dayside magnetosphere [Leonovich Mazur, 2016]. The potential well here is wide and shallow. The formation of standing waves along the radial coordinate becomes problematic.

Satellite observations show [Nishida, 1980], however, that plasma in the outer magnetosphere is present as plasmoids bounded along radial and azimuthal coordinates. The plasma density in a plasmoid may be an order of magnitude higher than in the vicinity of the outer magnetosphere. If such a plasmoid is located at a short distance from the plasmapause, it can cause a dramatic decrease in the Alfvén velocity from its maximum value at the outer edge at a short radial distance. In this case, the potential well becomes narrow and deep and makes the process of formation of radial standing waves more effective. The outer reflecting wall of the resonator will be located on the magnetic shell of the plasmoid.

One of the main features of such a 2D Alfvén wave resonator, which involves various plasmoids in the outer magnetosphere, is the lack of azimuthal symmetry. Characteristics of the resonator's eigenfrequencies for the plasmoids that are located at different distances from the plasmapause should vary. Depending on the azimuthal coordinate (local time) of the point of recording of initial oscillations, we are to observe stepwise changes in parameters of equidistant frequency groups, determined by the APCF processing method.

Return to the analysis of plots in Figure 3. If we compare the histograms of adjacent time intervals in Figure 3, $a, b$, we can see that all the peaks with identical numbers correspond completely to each other and have nearly identical $\Delta f$. In Figure 3, $a$, peaks 8 and 11 , for some reason, did not appear. They can, however, be added because they are pronounced in Figure $3 b$. The average gaps between the peaks for both the intervals coincide as well. In dimensionless units, $\delta \Delta f=4.0 \cdot 10^{-4}$. These coincidences suggest that in adjacent time intervals 12-13 and 13-14 LT equidistant groups of eigenfrequencies (6) have the same characteristics (7) and (8).

In the noon sector of the magnetosphere, the plasmapause shell number weakly depends on the azimuthal coordinate. Hence, in the right-hand side of (8) the Larmor radius $S$ and the period of the first harmonic at the outer edge $T_{1}$ is also independent of this coordinate. Variations in $\delta \Delta f$ with local time can be associated only with a variation in the resonator width $l_{x}$.

The above analysis of the histograms in Figure $3, a, b$ leads to an important conclusion. In the angular sector of azimuthal coordinates, which corresponds to the local time interval 12-14 LT, in the outer magnetosphere there is an almost ideal reflecting wall. Throughout the sector, it contains no discontinuities and is located at the same distance $l_{x}$ from the inner reflecting wall at the plasmapause. We can estimate this distance. In the vicinity of the outer edge of the plasmapause, the Larmor radius of radiation belt protons with an energy of 0.5 MeV S=200 km (Kovtyukh, Panasyuk, 2008), and the period of the first harmonic $T_{1}=40 \mathrm{~s}$ [Leonovich, Mazur, 2016]. Substituting these values in (8), in view of $\Delta t=0.1 \mathrm{~s}$ and $\delta \Delta f=4.0 \cdot 10^{-4}$, find $l_{x}=4000 \mathrm{~km}=0.60 R_{\mathrm{E}}$. The resulting value is larger than the plasmapause thickness, much smaller than the outer dayside magnetosphere, and is well suited for the radial size of the 2D Alfvén wave resonator.

Turn to the comparison of the histograms in Figure $3, a, c$. It is noticeable that the peaks with the same numbers have the $\Delta f$ values which are not connected with each other. The average gap between the peaks in the histogram presented in Figure 3, $c$ is much smaller: $\delta \Delta f=3.3 \cdot 10^{-4}$. The differences between the parameters of the peaks indicate that in the sector with the local time 14-15 LT there is an entirely different resonator with the radial size $l_{x}=0.73 R_{\mathrm{E}}$. In each histogram, we can see pronounced undistorted peaks. Such peaks should be formed by the entire signal time length rather than by its separate fragment. Hence, the transition from the peaks in the 13-14 LT sector to the peaks in the 1415 LT sector should occur in the vicinity of 14 LT for a very short time. Similarly, at a short angular distance the size of the resonator $l_{x}=0.60 R_{\mathrm{E}}$ in the $12-14 \mathrm{LT}$ sector is changed to $l_{x}=0.73 R_{\mathrm{E}}$ in the $14-15 \mathrm{LT}$ sector.

In the histogram in Figure 3, $d$, the average gap between the peaks is even less: $\delta \Delta f=2.9 \cdot 10^{-4}$. The radial size of the resonator increases to $l_{x}=0.83 R_{\mathrm{E}}$.

The overall picture of the azimuthal variations is as follows. In the 11-12 LT sector, the outer boundary of the resonator is located at a distance $l_{x}=0.83 R_{\mathrm{E}}$ from the inner boundary at the plasmapause. In the 12-14 LT sector, it jumps to the plasmapause for a distance $l_{x}=0.60 R_{\mathrm{E}}$; and in the $14-15 \mathrm{LT}$ sector, it again jumps away from it by $l_{x}=0.73 R_{\mathrm{E}}$.

Such a discontinuous azimuthal structure of the 2D Alfvén wave resonator can be considered direct evidence that in the radial direction its outer boundary is high-density plasmoids located in the outer magnetosphere. These plasmoids are separated from each other, have different angular sizes, and are located at different distances from the plasmapause along the azimuthal coordinate.

\section{CONCLUSION}

1. I have presented a modified upgrade version of the signal processing method. The method of the correlation function of amplitude and phase fluctuations is not related to the spectral analysis. It is based on a unique feature of APCF, which is derived from the influence of a sequence of special transformations on the original signal. The method allows us to detect the presence of an equidistant frequency group in the spectrum 
of the original signal and to measure the difference of two adjacent frequencies $\Delta f$ in such a group. The end product of the processing is a histogram of a set of $\Delta f$ values.

In the traditional spectrum, the presence of a peak at a certain frequency means that fluctuations of this frequency are a part of the original signal. In our spectrum (histogram), each peak corresponds not to one equidistant frequency but to a whole group of equidistant frequencies in the original signal. The position of the peak on the horizontal axis gives us not a separate frequency but the difference of two adjacent frequencies characteristic of the whole group.

2. I have examined the influence of noise that can occur in the original signal. It has been shown that even at a very high level of noise when its component in the spectrum completely absorbs and masks peaks of equidistant frequencies of the desired signal, the APCF method copes with the problem of detecting these frequencies. In the final histogram, we can see a distinct maximum in the $\Delta f$ value that has been found for the desired signal.

3. For the first time the APCF computer program has been applied to processing of real natural signals. As these signals I have used records of geomagnetic disturbances in the frequency range that corresponds to the high-frequency ULF range. I have processed four different intervals of a $1 \mathrm{hr}$ recording. For each interval, I have obtained a histogram of frequency differences $\Delta f$.

By comparing one of the histograms with the traditional spectrum (Figure 2), I have shown that the chaotic spectrum, which has always been considered as noise spectrum, actually has a well-ordered structure. It was found that most spectral peaks belong to one of the set (more than 10) of equidistant frequency groups. Each group has its own, distinct from other groups, difference of adjacent frequencies $\Delta f$. When combined in the total spectrum, peaks of these groups are superposed, involving not two and three groups of peaks but 11 ones. As a result, the spectrum has a very high density of peaks (Figure 2,a). The analysis of the mutual arrangement of these peaks on the frequency axis is much more complicated and does not allow us to identify any regularities. We just see their chaotic alternation.

4. The analysis of all the obtained histograms has revealed two characteristic features. In each of them there is a set (more than 10) of pronounced undistorted peaks. Most peaks on the $\Delta f$ axis are equidistant.

It has been shown that the equidistant frequency groups in the oscillation spectrum, which correspond to these peaks, are no other than allocated groups of eigenfrequencies of the 2D Alfvén wave resonator. The existence of such a resonator in the magnetosphere in the vicinity of the outer edge of the plasmapause has been predicted in theoretical studies [Guglielmi, Polyakov, 1983; Leonovich, Mazur, 1987]. The new processing method allowed us to experimentally confirm this prediction.

5. Equidistant peaks of each histogram feature an average gap between two adjacent peaks. The analysis of the local time dependence of these gaps provides powerful evidence that the outer boundary shell of the resonator is due to the presence of a background plasmoid on it. Such plasmoids have limited scales along the azimuthal coordinate and depending on this coordinate are located at different distances from the plasmapause, which coincides with the inner boundary of the resonator shell.

The work was performed with budgetary funding of Basic Research program II.16. The results were obtained using the equipment of Center for Common Use «Angara» http://ckp-rf.ru/ckp/3056.

\section{REFERENCES}

Gudzenko L.I. A statistical method for determining the characteristics of a noncontrolled self-oscillatory system. Izvestiya vuzov. Radiofizika [Radiophysics and Quantum Electronics]. 1962, vol. 5, no. 3, pp. 572-586. (In Russian).

Guglielmi A.V., Polyalov A.R. On the discreteness of spectrum of Alfvén oscillations. Geomagnetizm $i$ aeronomiya [Geomagnetism and Aeronomy]. 1983, vol. 23, iss. 2, pp. 341-343. (In Russian).

Kovtuykh A.S., Panasyuk M.I. Earth radiation belts. Plazmennaya geliogeofizika [Plasma Heliogeophysics] / eds. L.M. Zeleny, I.S. Veselovsky. Moscow, Fizmatlit Publ., 2008, vol. 1, pp. 510-534. (In Russian).

Leonovich A.S., Mazur V.A. Dynamics of small-scale Alfvén waves in magnetospheric resonator. Fizika plazmy [Plasma Phys.]. 1987, vol. 13, iss. 7, pp. 800-810. (In Russian).

Leonovich A.S., Mazur V.A. Lineinaya teoriya MGD-kolebanii magnitosfery. Moscow, Fizmatlit Publ., 2016, 480 p. (In Russian).

Nishida A. Geomagnetic diagnosis of the magnetosphere. Moscow, Mir Publ., 1980. 302 p. (In Russian). (English Edition: Geomagnetic Diagnosis of the Magnetosphere. Springer Science+Business Media, New York, 1978).

Polyakov A.R. New method of processing records of seismic oscillations based on analysis of correlation functions of random amplitude and phase fluctuations. Parts 1-2. Solnechno-zemnaya fizika [Solar-Terr. Phys.]. 2010, iss. 15, pp. 44-57. (In Russian).

Polyakov A.R. The structure of one-dimensional standing MHD waves in and at the boundary of the dayside plasmasphere. J. Atmos. Solar-Terr. Phys. 2014, vol. 119, pp. 193202.

Polyakov A.R. A method for detecting equidistant frequencies in the spectrum of a wideband signal. J. Atmos. Solar-Terr. Phys. 2017, vol. 152, pp. 30-40.

URL: http://ckp-rf.ru/ckp/3056 (accessed Jule 25, 2018).

How to cite this article

Polaykov A.R. Detecting groups of equidistant frequencies in spectra of geomagnetic pulsations. Solar-Terrestrial Physics. 2018. Vol. 4. Iss. 4. P. 33-41. DOI: 10.12737/stp-44201805. 